\title{
Renormalization group analysis for an asymmetric simple exclusion process
}

\author{
Sutapa Mukherji \\ Protein Chemistry and Technology, Central Food Technological Research Institute, Mysore -570 020, Karnataka
}

(Dated: July 7, 2021)

\begin{abstract}
A perturbative renormalization group method is used to obtain steady-state density profiles of a particle non-conserving asymmetric simple exclusion process. This method allows us to obtain a globally valid solution for the density profile without the asymptotic matching of bulk and boundary layer solutions. In addition, we show a nontrivial scaling of the boundary layer width with the system size close to specific phase boundaries.
\end{abstract}

Boundary layers are found to play an important role in boundary induced phase transitions of asymmetric simple exclusion processes (ASEPs). In ASEP, particles hop on a finite one dimensional lattice with a bias in a preferred direction. In addition, particles obey mutual exclusion principle that prohibits two particles from occupying the same lattice site [1-3]. In the steady state, the average particle distribution profile on the lattice depends completely on the particle injection rate, $\alpha$, at one end of the lattice and withdrawal rate, $\beta$, at the other end. Boundary layers appear in the particle distribution profile and, in general, their width, height, and location change sharply with the injection and withdrawal rates [4]. These rates are the tuning parameters for the boundary induced phase transitions which can be characterized through the shape and the properties of the density profile and, in particular, its boundary layer.

In order to obtain the steady-state density profile of the ASEP in the hydrodynamic limit, one has to solve a boundary value problem involving singular nonlinear differential equation(s) for the particle density profile with boundary conditions given by the boundary rates [4, 5]. The singularity in the equation is due to the highest order derivative term that appears along with a small multiplicative paramater, $\epsilon$, which is inversely proportional to the system size, $N$. Solutions of such equations have boundary layers which are narrow segments over which the solution varies rapidly in comparison with the remaining, slowly varying (bulk) part of the solution . Here by "boundary layer', we imply a solution of the differential equation under a specific limit. This solution need not necessarily appear only near the system boundary. In the same spirit, the rest of the density profile is referred as the bulk solution which also need not be confined to the interior of the system. In particular, in ASEP, the bulk solution often satisfies one of the boundary conditions. Methods of boundary layer analysis can be used to find a uniform approximation for the solution of the boundary value problem. This is achieved by first constructing the asymptotic expansions for the solutions near the boundary-layer and the bulk regions and then joining these solutions by matching them in the appropriate limit. A more general method to obtain the density profile including its boundary layers involves obtaining the fixed points of the boundary layer differential equation [6]. Since the boundary layer saturates to the bulk solution, one can extract information about the bulk solution by finding out the boundary layer fixed points and their stability properties.

Although boundary layer based methods are useful in many cases, the scaling behavior of the width of the boundary layer with the system size may not be fully uncovered through this method. Previous boundary-layer based work shows that the scaling of the boundary layer width follows simple dimensional analysis. This, however, need not be the case always, since Monte-Carlo simulations on certain ASEP models show deviations from simple dimensional analysis [7]. Historically, renormalization group (RG) analysis appears to be one of the efficient tools that can be used in situations where simple dimensional considerations do not work [8]. The application of RG analysis to singularly perturbed differential equations shows that the presence of multiple scales in the problem comes out as a natural consequence of the analysis and no apriori knowledge is required for obtaining multiple scales [9]. The utility of the renormalisation group approach in solving various singular perturbation problems has been further elaborated later in [10]. In view of this, a natural question arises as whether $\mathrm{RG}$ analysis can reveal the presence of multiple scales, if any, in ASEP.

The main purpose of the paper is to present an RG analysis for the ASEP described here and attempt to find out if there exists any hidden scaling not seen so far through the boundary layer method. As we shall show below, this analysis indeed shows the possibility of a different scaling near certain phase boundaries of ASEP. Apart from this, RG has additional benefits over conventional boundary layer methods [9, 11]. It allows us to obtain uniform, globally valid solutions without the need of any asymptotic matching as done in boundarylayer methods. This simple ASEP model demonstrates how this goal is achieved. Similar to the boundary layer method, RG analysis starts with a naive perturbation expansion of the boundary layer equation. In both methods, the future steps rely on finding the the analytical solution of the differential equation at $O\left(\epsilon^{0}\right)$ level; a task which may not be feasible for complex ASEP models. However, in case of RG, this difficulty of finding an exact solution of the lowest order equation may be bypassed by guessing a naive solution. Although, this gives rise to certain ambiguities, RG is believed to be robust against 
such pitfalls [11]. In view of this, we hope that these studies on the simplest ASEP model may further be extended to more complex processes.

The specific model that we consider here is totally asymmetric in the sense that particles move only in one direction along a lattice of $N$ sites provided the target site is empty. In addition, we also consider particle nonconserving processes which include particle evaporation (adsorption) from (to) an occupied (unoccupied) lattice site. In the discrete picture, the dynamics of the particle can be described in terms of a variable $\tau_{i}$ that denotes the particle occupancy of the $i$ th site. This variable can have values $\tau_{i}=1$ or 0 if the $i$ th site is occupied or empty, respectively. The time evolution of the variable $\tau_{i}$ can be expressed as

$$
\frac{d \tau_{i}}{d t}=\tau_{i-1}\left(1-\tau_{i}\right)-\tau_{i}\left(1-\tau_{i+1}\right)+\omega_{a}\left(1-\tau_{i}\right)-\omega_{d} \tau_{i},(1)
$$

where the first two terms on the right hand side of the equation arise from particle hopping to the neighboring site and the last two terms are the gain and loss terms due to particle adsorption and evaporation at rates $\omega_{a}$ and $\omega_{d}$, respectively. We shall consider a simple continuum mean-field description which requires first a statstical averaging of the time evolution equation with the approximation $\left\langle\tau_{i} \tau_{j}\right\rangle=\left\langle\tau_{i}\right\rangle\left\langle\tau_{j}\right\rangle$ and then going over to a continuum limit that involves $N \rightarrow \infty$, lattice spacing $a \rightarrow 0$ limits with $N a$ remaining finite. For simplicity, we choose $N a=1$ in the following.

In the continuum limit, the steady state $\left(\frac{d \rho}{d t}=0\right)$ particle density, $\rho(x)$, at position $x$, satisfies the differential equation

$$
\epsilon \frac{d^{2} \rho}{d x^{2}}+(2 \rho-1) \frac{d \rho}{d \tilde{x}}+\Omega(1-2 \rho)=0 .
$$

where $\epsilon=1 /(2 N)$. First two terms in this equation can be expressed as the gradient of the current $-\frac{\partial J}{\partial x}$, with $J=-\epsilon \frac{\partial \rho}{\partial x}+\rho(1-\rho)$. The third term appears due to particle loss and gain at equal rates $\left(\omega_{a}=\omega_{d}\right)$ with $\Omega=\omega_{d} N$. In order to obtain the steady-state density profile, one needs to solve equation (2) along with the boundary conditions $\rho(x=0)=\alpha$ and $\rho(x=1)=\gamma=1-\beta$ at the two ends of the lattice. The particle conserving model has been solved exactly [2, 3] and it appears that the phase diagram broadly consists of three phases with average bulk particle density remaining constant in each phase. For $\alpha<1 / 2$ and $\beta>\alpha$, the system is in a low density phase where the average particle density in the bulk of the profile is constant at a value $\rho=\alpha(<1 / 2)$. The bulk density profile satisfies the boundary condition at $x=0$ and a boundary layer appearing near $x=1$ satisfies the boundary condition at $x=1$. For $\beta<1 / 2$ and $\alpha>\beta$, the system is in a high density phase where the average bulk density is $\rho=1-\beta>1 / 2$. Since in this case, the constant bulk profile satisfies the boundary condition at $x=1$, the boundary condition at $x=0$ is satisfied by a boundary layer. For $\alpha, \beta>1 / 2$, the average particle density is constant at a value $\rho=1 / 2$ with boundary layers appearing at both ends of the system. Since the particle current has the maximum value in this phase, this phase is known as the maximum current phase. In the particle non conserving model, the bulk density is not constant but there exist similar lowdensity $(\rho<1 / 2$ in the bulk), high-density $(\rho>1 / 2$ in the bulk) and maximum current $(\rho=$ constant $=1 / 2)$ phases in addition to several other phases where the density profile has constant, maximum-current parts along with nonconstant parts similar to those in high or lowdensity phases (see figure 1) [4, 7, 12]. As we shall show below, the approach to the HM or LM phase from the high-density or low-density phases respectively is special since, very close to the phase boundary, the boundarylayer width is expected to scale with the system size as $N^{-1 / 2}$ unlike its usual scaling as $N^{-1}$.

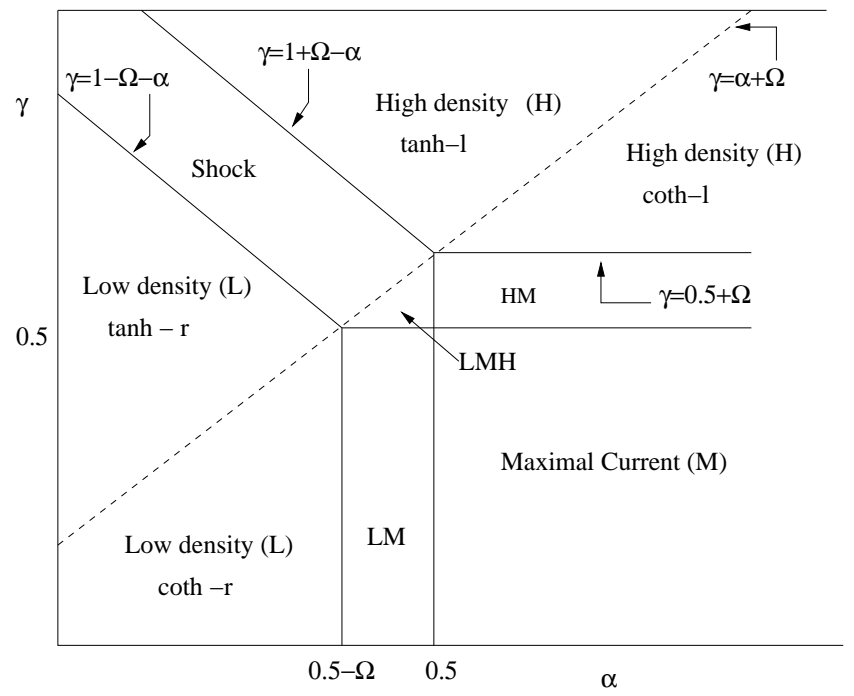

FIG. 1: The phase diagram of the particle non-conserving totally asymmetric simple exclusion process with $\omega_{a}=\omega_{d}$. HM and LM denote high-density-maximal-current and lowdensity-maximal-current phases where the density profile has a linear part (similar to high-density or low-density phases, see equation (3)) along with a constant profile (similar to the maximal current phase). In a similar way, LMH denotes a phase where the density profile has parts similar to that of low-density (L), high-density $(\mathrm{H})$ and maximal (M) current phases.

The boundary layer analysis for this totally asymmetric simple exclusion process proceeds as follows. For very small $\epsilon$, one may ignore the second-order derivative term in (2) and obtain a solution of the corresponding first order equation as

$$
\rho_{o}(x)=\Omega x+c
$$

This, being the zeroth order solution of the original equation, is expected to describe most of the density profile. Since in the boundary layer language, this solution is 
known as the outer solution, we have denoted this solution as $\rho_{o}(x)$. Clearly, a solution of a first order equation cannot satisfy two boundary conditions and hence, the presence of a boundary layer becomes necessary. A boundary layer solution for such a second order differential equation is subjected to two conditions. For example, in the low or high density phase, the boundary layer satisfies the boundary condition at one end and merges to the bulk solution (outer solution) in the other limit. In order to satisfy two conditions, the second derivative term of (2) becomes necessary for the description of the boundary layer. A dominant balance argument shows that for a boundary layer near $x \sim O(0)$, one requires a rescaling of $x$ as $\tilde{x}=\frac{x}{\epsilon}$. Considering the fact that there can be various possible locations of the boundary layer, for example, near $x=0$ or $x=1$ boundary or in the interior of the lattice, we reexpress equation (2) in terms of a scaled variable $\tilde{x}=\frac{\left(x-x_{0}\right)}{\epsilon}$, where $x_{0}$ indicates the location of the boundary layer. For example, for a boundary layer located near $x=1$ boundary, we have $x_{0}=1$. The differential equation in terms of $\tilde{x}$ is

$$
\frac{d^{2} \phi}{d \tilde{x}^{2}}+\phi \frac{d \phi}{d \tilde{x}}-2 \Omega \epsilon \phi=0,
$$

where $\phi=2 \rho-1$. Ignoring the last term in the $\epsilon \rightarrow 0$ limit, we have the zeroth order boundary layer equation

$$
\frac{d^{2} \phi}{d \tilde{x}^{2}}+\phi \frac{d \phi}{d \tilde{x}}=0
$$

which upon one integration appears as

$$
\frac{d \phi}{d \tilde{x}}+\frac{\phi^{2}}{2}=c_{1}
$$

where $c_{1}$ is the integration constant. Since, the boundary layer is expected to saturate to the bulk solution (outer solution) in the appropriate limit of $\tilde{x}$, we consider $c_{1}=$ $\frac{\phi_{b}^{2}}{2}$, where $\phi_{b}=2 \rho_{b}-1$ with $\rho_{b}$ as the corresponding bulk density at the saturation edge. Since the solution of the boundary layer equation (6) is usually called the inner solution, we denote this solution as $\phi_{\mathrm{in}}$. The explicit solutions of (6) are

$$
\begin{array}{r}
\phi_{\text {in }}=\phi_{b} \tanh \left[\frac{\phi_{b}}{2}\left(\tilde{x}+c_{2}\right)\right] \text { and } \\
\phi_{\text {in }}=\phi_{b} \operatorname{coth}\left[\frac{\phi_{b}}{2}\left(\tilde{x}+c_{2}\right)\right],
\end{array}
$$

where $c_{2}$ is the second integration constant. In terms of $\rho$, the boundary layer or inner solutions are

$$
\begin{array}{r}
\rho_{\text {in }}=\frac{1}{2}+\left(1 / 2-\rho_{b}\right) \tanh \left[\frac{\left(1-2 \rho_{b}\right)}{2}\left(\tilde{x}+c_{2}\right)\right] \text { and } \\
\rho_{\text {in }}=\frac{1}{2}+\left(1 / 2-\rho_{b}\right) \operatorname{coth}\left[\frac{\left(1-2 \rho_{b}\right)}{2}\left(\tilde{x}+c_{2}\right)\right] .
\end{array}
$$

The saturation of tanh and coth functions for large values of their arguments is responsible for saturation of the boundary layers to the bulk. In order to understand how various conditions are satisfied by the boundary layer, let us consider the density profile of the lowdensity phase as an example. The boundary layer located near $x=1$ boundary satisfies the boundary condition at $x=1$ and saturates to the bulk solution in the $\tilde{x} \rightarrow-\infty(x<1)$ limit. Such a boundary layer can be consistently described by equation (9) since $\rho_{\text {in }}$ approaches $\rho_{b}$ in the $\tilde{x} \rightarrow-\infty$ limit. The matching (saturation) with the outer solution in equation (3) demands $\rho_{o}(x=1)=\rho_{b}=\rho_{\text {in }}(\tilde{x} \rightarrow-\infty)$.

The RG analysis starts with the perturbative expansion for the boundary layer solution. The renormalized perturbative theory, however, gives the uniform globally valid solution for the entire density profile. In order to find the solution of the boundary layer equation (4) perturbatively, we assume a naive expansion of the solution as $\phi(\tilde{x})=\phi_{0}(\tilde{x})+\epsilon \phi_{1}(\tilde{x})+\epsilon^{2} \phi_{2}(\tilde{x})+\ldots .$. While at $O\left(\epsilon^{0}\right)$, $\phi_{0}$ satisfies the same equation as ([6), at $O(\epsilon)$, we have

$$
\frac{d^{2} \phi_{1}}{d \tilde{x}^{2}}+\frac{d\left(\phi_{0} \phi_{1}\right)}{d \tilde{x}}-2 \Omega \phi_{0}=0
$$

We rewrite the solution of the $O\left(\epsilon^{0}\right)$ equation as

$$
\begin{aligned}
& \phi_{0}=\sqrt{c} \operatorname{coth}\left[\frac{\sqrt{c}}{2}(\tilde{x}+k)\right], \\
& \phi_{0}=\sqrt{c} \tanh \left[\frac{\sqrt{c}}{2}(\tilde{x}+k)\right],
\end{aligned}
$$

where $c$ and $k$ are the integration constant with $c=\phi_{b}^{2}$. In order to find $\phi_{1}$, we use the tanh solution for $\phi_{0}$ in (11). The solution for $\phi_{1}$ is

$$
\begin{aligned}
& \phi_{1}=(2 p)^{-1} \operatorname{sech}\left[\frac{1}{2} p(k+\tilde{x})\right]^{2}[2 p \Omega(k+\tilde{x})- \\
& \Omega p^{2}(k+\tilde{x})^{2}+(k+\tilde{x}) p \bar{c}+2 p \bar{k}- \\
& 4 \Omega p(k+\tilde{x})\left\{\log \left[1+e^{-p(k+\tilde{x})}\right]+\right. \\
& \left.\log \left[\cosh \left\{\frac{p}{2}(k+\tilde{x})\right\}\right]\right\}+4 \Omega \operatorname{Li}_{2}(\exp [-p(k+\tilde{x})])+ \\
& \left(-2 \Omega+\bar{c}+4 \Omega \log \left[\cosh \left\{\frac{1}{2} p(k+\tilde{x})\right\}\right]\right) \times \\
& \sinh [p(k+\tilde{x})]]
\end{aligned}
$$

where $p=\sqrt{c}, \bar{c}$ and $\bar{k}$ are constants of integration and $\mathrm{Li}_{n}(y)$ is the Polylogarithm function of order $n$ and argument $y$. In the boundary layer regime, the Polylogarithm function is expected to have real values. For example, for the tanh boundary layer present near $x=1$ in the low density phase, the argument of the exponential function in the Polylogarithm is always large negative $\left(\tilde{x} \rightarrow-\infty\right.$ and $\left.p=\phi_{b}=2 \rho_{b}-1<0\right)$. This makes the argument of Polylogarithm negligibly small. The naive perturbation done here breaks down due to the term $4 \Omega(2 p)^{-1} \operatorname{sech}\left[\frac{1}{2} p(k+\tilde{x})\right]^{2} \sinh [p(k+\tilde{x})] \log \left[\cosh \left\{\frac{1}{2} p(k+\right.\right.$ 
$\tilde{x})\}]$ that diverges as $\tilde{x} \rightarrow \infty$. This term, when multiplied with $\epsilon$, becomes comparable to the zeroth order solution since $\epsilon \tilde{x}$ becomes $O(1)$ in the $\tilde{x} \rightarrow \pm \infty$ limit. The appearance of this divergence in the naive perturbation theory is the manifestation of the singularity in the problem.

The perturbative solution upto $O(\epsilon)$ can be expressed as

$$
\begin{aligned}
& \phi(\tilde{x})=p \tanh \left[\frac{p}{2}(k+\tilde{x})\right]+\epsilon \mathcal{R}(\tilde{x})+ \\
& \epsilon \frac{4 \Omega}{p} \log \left[\cosh \left\{\frac{1}{2} p(k+x)\right\}\right] \tanh [p(k+x) / 2],
\end{aligned}
$$

where the secular term is written clearly and all the regular terms are represented together by $\mathcal{R}$. Considering the the $\tilde{x} \rightarrow \infty$ limit, in which $\log \left[\cosh \left[\frac{1}{2} p(k+\tilde{x})\right] \sim\right.$ $\frac{1}{2} p(k+\tilde{x})$, we separate the divergence in the perturbation series as

$$
\begin{aligned}
& \phi(\tilde{x})=p \tanh \left[\frac{p}{2}(k+\tilde{x})\right]+\epsilon \mathcal{R}(\tilde{x})+ \\
& \epsilon 2 \Omega(k+\tilde{x}-\mu) \tanh \left[\frac{p}{2}(k+\tilde{x})\right]+ \\
& \epsilon 2 \Omega \mu \tanh \left[\frac{p}{2}(k+\tilde{x})\right],
\end{aligned}
$$

where $\mu$ is an arbitrary length scale chosen in such a way that the second term is now no more diverging and the entire divergence is contained in the last term. The divergence is absorbed by introducing a renormalized constant, $p_{r}$, as $p=p_{r}(\mu)+Z_{1}(\mu)$ where $Z_{1}(\mu)=$ $a_{1} \epsilon+a_{2} \epsilon^{2} \ldots$. The divergence in (16) can be absorbed if $a_{1}=-2 \Omega \mu$. The renormalized perturbation series for $\phi$ is now

$$
\begin{aligned}
& \phi=p_{r}(\mu) \tanh \left[\frac{p_{r}(\mu)}{2}(k+\tilde{x})\right]+\epsilon 2 \Omega(k+\tilde{x}-\mu) \times \\
& \tanh \left[\frac{p_{r}(\mu)}{2}(k+\tilde{x})\right] \\
& -\epsilon \Omega \mu p_{r}(\mu)(k+\tilde{x}) \operatorname{sech}\left[\frac{p_{r}(\mu)}{2}(k+\tilde{x})\right]^{2}+\epsilon \mathcal{R}(\tilde{x}),(1
\end{aligned}
$$

where the last term arises from the expansion of the tanh term in small $\epsilon$. The renormalized theory cannot depend on an arbitrary length scale $\mu$. The condition, $\frac{\partial \phi}{\partial \mu}=0$, leads to the following equation

$$
\begin{aligned}
& \frac{\partial \phi}{\partial \mu}=\frac{d p_{r}}{d \mu} \tanh \left[\frac{p_{r}}{2}(k+\tilde{x})\right]+p_{r} \frac{d p_{r}}{d \mu} \frac{(k+\tilde{x})}{2} \times \\
& \operatorname{sech}^{2}\left[\frac{p_{r}}{2}(k+\tilde{x})\right]-\epsilon \Omega p_{r}(k+\tilde{x}) \operatorname{sech}^{2}\left[\frac{p_{r}}{2}(k+\tilde{x})\right]- \\
& \epsilon \Omega \mu(k+\tilde{x}) \operatorname{sech}^{2}\left[\frac{p_{r}}{2}(k+\tilde{x})\right] \frac{d p_{r}}{d \mu}+\epsilon \Omega \mu(k+\tilde{x})^{2} \times \\
& \operatorname{sech}^{2}\left[\frac{p_{r}}{2}(k+\tilde{x})\right] \tanh \left[\frac{p_{r}}{2}(k+\tilde{x})\right] \frac{d p_{r}}{d \mu} \\
& -2 \Omega \epsilon \tanh \left[\frac{p_{r}}{2}(k+\tilde{x})\right]+2 \Omega \epsilon(k+\tilde{x}-\mu) \frac{(k+\tilde{x})}{2} \times \\
& \operatorname{sech}^{2}\left[\frac{p_{r}}{2}(k+\tilde{x})\right] \frac{d p_{r}}{d \mu}=0
\end{aligned}
$$

Up to $O(\epsilon)$, this leads to an RG equation

$$
\frac{d p_{r}}{d \mu}=\epsilon(2 \Omega) \text {. }
$$

Substituting the solution for, $p_{r}(\mu)$, as $p_{r}(\mu) \sim 2 \Omega \mu \epsilon+$ $C_{p}$ in (17), and setting $\mu=\tilde{x}$, we have the following renormalized profile

$$
\begin{aligned}
& \phi(x)=\left(C_{p}+2 \Omega \epsilon \tilde{x}\right) \tanh \left[\left(C_{p}+2 \Omega \epsilon \tilde{x}\right)(k+\tilde{x}) / 2\right]+ \\
& \epsilon\left[2 \Omega k \tanh \left[\left(C_{p}+2 \Omega \epsilon \tilde{x}\right)(k+\tilde{x}) / 2\right]-\right. \\
& \left.\tilde{x}(k+\tilde{x}) \Omega C_{p} \operatorname{sech}^{2}\left[\left(C_{p}+2 \Omega \epsilon \tilde{x}\right)(k+\tilde{x}) / 2\right]\right] \\
& +\epsilon \mathcal{R}(\tilde{x})
\end{aligned}
$$

where $C_{p}$ is the integration constant. This solution is the globally valid solution that has a boundary layer of tanh kind near $\tilde{x} \sim 0$ and a linear profile as in (3) for large $\tilde{x}$ limit $(+\infty$ or $-\infty)$ where tanh saturates. The first term in (20) approximates to a linear solution for $\tilde{x} \rightarrow \pm \infty$ limit. For small $\tilde{x}$, i.e. near the boundary, the first term leads to a tanh type boundary layer (neglecting the $\tilde{x}$ dependent term in the prefactor of the tanh term). The second term, due to its prefactor, $\epsilon$ has a negligible contribution for large $\tilde{x}$ as well as $\tilde{x} \approx 0$. In a similar way, the third term also has negligible contribution in the $\tilde{x} \rightarrow \pm \infty$ (due to the sech term) and for $\tilde{x} \approx 0$.

In the high-density phase, for certain parameter values, the density profile has a tanh type boundary layer near $x=0$ boundary. In the $\tilde{x} \rightarrow \infty$ limit, the boundary layer merges to a linear profile satisfying the boundary condition at $x=1$. The integration constants of (20) determined using the boundary conditions $\phi(\tilde{x}=0)=$ $2 \alpha-1$ and $\phi(x=1)=2 \gamma-1$ are found as

$$
C_{p} k=\ln \frac{\gamma+\alpha-\Omega-1}{\gamma-\alpha-\Omega} \text { with } C_{p}=2 \gamma-2 \Omega-1 .
$$

In the low density phase, the linear part of the density profile satisfies the boundary condition at $x=0$ and the tanh type boundary layer satisfies the boundary condition at $x=1$. Thus, imposing the condition $\phi(\tilde{x}=0)=2 \gamma-1$ and $\phi(x=0)=2 \alpha-1$, we have

$$
C_{p} k=\ln \frac{\alpha+\Omega+\gamma-1}{\alpha+\Omega-\gamma} \text { with } C_{p}=2 \Omega+2 \alpha-1 .
$$

The renormalized density profile with a coth type zeroth order solution can be derived in an identical way. At the lowest order, the density profile has the form

$$
\phi=\left(C_{p}+2 \Omega \epsilon \tilde{x}\right) \operatorname{coth}\left[\left(C_{p}+2 \Omega \epsilon \tilde{x}\right)(k+\tilde{x}) / 2\right] .
$$

In the high density phase, for certain range of parameter values, one may have a coth boundary layer satisfying the boundary condition at $x=0$. In this case, the constants in the renormalised profile are

$$
C_{p}=2 \gamma-2 \Omega-1, \quad C_{p} k=\ln \frac{\gamma-\Omega+\alpha-1}{\alpha-\gamma+\Omega} .
$$


Density profiles of this shape is observed in the highdensity phase, on the right side of the line $\gamma=\alpha+\Omega$ (see figure 11). Further, as one proceeds near its phase boundary, $\gamma=1 / 2+\Omega$, demarcating the high-density and high-density-maximum current (HM) phase, the new scaling of the boundary-layer width with the system size $N$ as $N^{-1 / 2}$, takes over. Similar scaling behavior of the boundary layer width is seen as one approaches the phase boundary $\alpha=1 / 2-\Omega$ between the low-density and the LM phase from the low density side.

In conclusion, we have presented a perturbative renormaization group analysis for a particle non conserving totally asymmetric simple exclusion process. Such systems with open boundaries are found to exhibit boundary driven phase transitions in the steady state. The shape of the average particle density profiles in various phases depends crucially on the boundary parameters which, in this case, are the particle withdrawal and injection rates at the boundaries. In various phases, particle density profiles, in general, have rapidly varying, narrow boundary layer parts and slowly varying bulk parts. A complete solution for the density profile can be obtained by using the method of boundary layer analysis which provides a systematic tool to solve the singular differential equation which, in the present case, describes the steady state particle density profile. The full density profile is obtained by finding the boundary layer and the bulk solutions of the singular differential equation and matching these two solutions in appropriate limits. Here we present a renormalisation group analysis of the singular differential equation and show that through this method one can arrive at a global solution of the differential equation without any asymptotic matching of the boundary layer and the bulk solutions. The globally valid, general solution, thus obtained, contains in itself both the boundary layer and the bulk parts which can be individually identified by considering different limits of the general solution. Further, it is shown that as the system approaches the low-density-maximal current phase or the high-density-maximal current phase from the lowdensity or high-density sides, respectively, the boundary layer width scales as $N^{-1 / 2}$ rather than $N^{-1}$.

Acknowledgement I thank S. M. Bhattacharjee for useful discussions. Financial support from the Department of Science and Technology, India and warm hospitality of ICTP (Italy), where the work was initiated, are gratefully acknowledged.
[1] T. Liggett, Interacting Particle Systems: Contact, Voter and Exclusion Processes (Springer-Verlag, Berlin, 1999).

[2] B. Derrida, M. R. Evans, V. Hakim and V. Pasquier, J. Phys. A 26, 1493 (1993).

[3] G. Schuetz and E. Domany, J. Stat. Phys. 72, 277 (1993).

[4] S. Mukherji and S. M. Bhattacharjee, J. Phys. A 38, L285 (2005); S. Mukherji and V. Mishra, Phys. Rev. E 74, 01116 (2006).

[5] J. D. Cole, Perturbation Methods in Applied Math- ematics (Blasidal Publishing company, Walthum, MA: 1968).

[6] S. Mukherji, Phys. Rev. E 79, 041140 (2009).

[7] A. Parmeggiani, T. Franosch and E. Frey, Phys. Rev. E
70046101 (2004); see also 12].

[8] D. J. Amit, Field Theory, the renormalisation Group and Critical Phenomena (World Scientific, Singapore, 1984).

[9] Lin-Yuan Chen, Nigel Goldenfeld and Y. Oono, Phys. Rev. E 54, 376 (1996)

[10] R. E. O'Mallet, Jr. and E. Kirkinis, Studies in applied mathematics, Vol 124 (2010).

[11] John Veysey II and Nigel Goldenfeld, Rev. Mod. Phys.79, 883 (2007).

[12] M. R. Evans, R. Juhasz and L. Santen Phys. Rev. E 68 026117 (2003). 